\title{
Enhanced tumor radiosensitivity by a survivin dominant-negative mutant
}

\author{
QING-ZHONG YUAN ${ }^{1,2^{*}}$, CHUN-TING WANG $^{1}$, YONG-QIU MAO ${ }^{1}$, PENG ZHANG $^{3 *}$, \\ HUA-SHAN SHI ${ }^{1 *}$, ZHI-YONG LI ${ }^{1}$, LI PAN $^{1 *}$, DAN-DAN YU ${ }^{1}$, FEI LENG ${ }^{1}$, XIANG CHEN $^{1}$, \\ WEI YING ${ }^{3}$, JING-HUI XU ${ }^{3}$, WEI LI ${ }^{3}$, FAN WU $^{3}$, YUAN WEN ${ }^{1}$, TIAN-TAI MA ${ }^{1}$ and YU-QUAN WEI ${ }^{1}$ \\ ${ }^{1}$ State Key Laboratory of Biotherapy and Cancer Center, West China Hospital, West China Medical School, \\ Sichuan University, Keyuan Road 4, Chengdu, Sichuan; ${ }^{2}$ Department of Oncology, No. 313 Hospital of PLA, \\ Huludao, Liaoning; ${ }^{3}$ Department of Radiation Oncology, Sichuan Cancer Hospital, Chengdu, Sichuan, P.R. China
}

Received April 28, 2009; Accepted June 19, 2009

DOI: 10.3892/or_00000610

\begin{abstract}
Radiosensitivity of tumors is due to a complex interaction of various factors, it has been reported that survivin also acts as a constitutive and inducible radioresistance factor in a panel of tumor cells and approaches designed to inhibit survivin expression or function may lead to tumor sensitisation to chemical and physical agents. Previously, we found that the plasmid encoding the phosphorylation-defective mouse survivin threonine $34 \rightarrow$ alanine mutant complexed to DOTAPchol liposome (Lip-mS) can suppress murine primary breast carcinoma. However, little is known regarding the biological effect of Lip-mS combined with radiation. The present study was designed to determine whether Lip-mS could enhance the anti-tumor activity of radiation. The Lewis Lung Carcinoma (LLC) cells treated with a combination of Lip-mS and radiation displayed apparently increased apoptosis compared with those treated with Lip-mS or radiation alone. Mice bearing LLC tumors were treated with intravenous injections of Lip-mS and radiation, the combined treatment significantly reduced mean tumor volume compared with either treatment alone. Moreover, the anti-tumor effect of Lip-mS combined with radiation was greater than their additive effect when compared with the expected effect of the combined treatment. These data suggest that inhibition of survivin using a dominantnegative mutant, survivin T34A, could sensitize LLC cells to radiation efficiently and the synergistic anti-tumor activity may in part result from increasing the apoptosis of tumor cells,
\end{abstract}

Correspondence to: Dr Chun-Ting Wang, National Key Laboratory of Biotherapy and Cancer Center, West China Hospital, West China Medical School, Sichuan University, Keyuan Road 4, Chengdu, Sichuan, P.R. China

E-mail: yuquanwei@hotmail.com

*Contributed equally

Key words: survivin, radiosensitivity, tumor, apoptosis, antiangiogenic therapy inhibiting tumor angiogenesis and inducing a tumor-protective immune response in the combined treatment.

\section{Introduction}

As the smallest member of the inhibitors of apoptosis protein (IAP) family, survivin is highly expressed in most common neoplasms, but is generally absent or low in most differentiated normal tissues (1-3). Moreover, the overexpression of survivin has been correlated with an enhanced proliferative index, reduced levels of apoptosis, increased rate of tumor recurrence, decreased overall survival and appears to be involved in tumor cell resistance to ionizing radiation (4) and some anticancer agents $(5,6)$.

Due to its dual role in suppressing apoptosis and regulating cell division, survivin is vital for cancer cell survival, which makes it an attractive target for new anticancer interventions $(7,8)$. In recent years, considerable efforts including antisense oligonucleotides (9), hammerhead ribozymes (10), RNA interference $(11,12)$, cancer vaccines (13) and dominant-negative mutants (14) have been made to counteract survivin and several preclinical studies have already demonstrated that down-regulation of survivin expression or function could inhibit tumor growth potential, increase spontaneous and induced apoptosis and sensitize tumor cells to anti-cancer agents and irradiation.

It was reported that one of the dominant-negative mutants, survivin Thr34 $\rightarrow$ Ala, which abolished a phosphorylation site for cdc2-cyclin B1 and prevented survivin binding to activated caspase-9 (15), could reduce tumor cell proliferative potential and lead to caspase-dependent apoptosis in melanoma cell lines (14). Previously, we also found that the plasmid encoding the phosphorylation-defective mouse survivin threonine $34 \rightarrow$ alanine mutant complexed to DOTAP-chol liposome (Lip-mS) can suppress murine highly metastatic breast carcinoma (16), however, little is known regarding the biological effect of Lip-mS combined with radiation, so the present study was designed to determine whether Lip-mS could enhance the anti-tumor activity of radiation and to explore the possible mechanisms of interaction between survivin targeting agents and radiation. 


\section{Materials and methods}

Cell line and culture conditions. The Lewis Lung Carcinoma (LLC) cell line of C57BL/6 mouse origin was purchased from the American Type Culture Collection (ATCC, Rockville, MD), cultured in DMEM (Gibco BRL, Grand Island, NY) and supplemented with $10 \%$ heat-inactivated fetal bovine serum (FBS) and maintained in a humidified incubator at $37^{\circ} \mathrm{C}$ in $5 \%$ $\mathrm{CO}_{2}$ atmosphere.

Plasmid DNA preparation. The recombinant plasmid encoding the phosphorylation-defective mouse survivin threonine $34 \rightarrow$ alanine mutant (mS-T34A) was purchased from Invivogen Corporation and confirmed by restriction endonuclease analysis, PCR and DNA sequence analysis. The plasmid was prepared using Endofree Plasmid Giga kit (Qiagen, Chatsworth, CA). Endotoxin levels of the plasmid DNA prepared were determined by Tachypleus Amebocyte Lysate (TAL). No genomic DNA, small DNA fragments, or RNA were detected in the DNA prepared and the $\mathrm{OD}_{260 / 2} 80$ ratios of the plasmid DNA prepared were between 1.8-2.0. The DNA was eventually dissolved in sterile endotoxin-free water and stored at $-20^{\circ} \mathrm{C}$ before use.

Liposome preparation. DOTAP-chol liposome was prepared using the procedure described previously (17). Briefly, the cationic lipid DOTAP was mixed with the neutral lipid chol at equimolar concentrations. The mixed lipids were dissolved in chloroform in a $100 \mathrm{ml}$ round bottomed flask. Then, the clear solution was rotated on a Buchi rotary evaporator at $30^{\circ} \mathrm{C}$ for $30 \mathrm{~min}$ to make a thin film, the flask containing the thin lipid film was dried under vacuum for $15 \mathrm{~min}$. The film was hydrated in $5 \%$ dextrose in water (D5W) to give a final concentration of $7 \mathrm{mM}$ DOTAP and $7 \mathrm{mM}$ chol, referred to as $7 \mathrm{mM}$ DOTAP:chol. The hydrated lipid film was rotated in a water bath at $50^{\circ} \mathrm{C}$ for $45 \mathrm{~min}$ and then $35^{\circ} \mathrm{C}$ for $10 \mathrm{~min}$. The mixture was allowed to stand in the parafilm-covered flask at room temperature overnight, after which the mixture was sonicated at low frequency for $5 \mathrm{~min}$ at $50^{\circ} \mathrm{C}$, transferred to a tube and heated for $10 \mathrm{~min}$ at $50^{\circ} \mathrm{C}$. The mixture was sequentially extruded through Millipore (Billerica, MA) polycarbonate membrane of decreasing size: $0.2 \mu \mathrm{m}$ for 5 times and $0.1 \mu \mathrm{m}$ for 3 times using syringes. Liposome were stored under argon gas at $4^{\circ} \mathrm{C}$. DOTAP was purchased from Avanti Polar Lipids (Alabaster, AL) and highly purified chol was purchased from Sigma (St. Louis, MO).

Preparation of DOTAP-chol liposomelplasmid DNA. DNA:liposome mixtures were prepared in accordance with a previously described method (18). Briefly, DOTAP-chol $(20 \mathrm{mM})$ and plasmid DNA stock solution diluted in 5\% dextrose in water (D5W) were mixed in equal volumes to give a final concentration of $4 \mathrm{mM}$ DOTAP-chol, i.e., $150 \mu \mathrm{g}$ DNA in $300 \mu \mathrm{l}$ final volume (ratio, 1:2.6). These reagents were diluted and mixed at room temperature. The DNA solution was added to DOTAP-chol liposome and rapidly mixed by pipetting up and down two times with the pipette tip. The DNA:liposome mixture thus prepared was precipitate-free and used for all the in vivo experiments. The size of the DNA fragments in the DNA:liposome mixture was determined to be in the range of $300-325 \mathrm{~nm}$.

Radiation. Cells and mice were irradiated with $\mathrm{Co}^{60} \gamma$-rays at a dose rate of $92 \mathrm{cGy} / \mathrm{min}$ (Nuclear Power Institute of China, Sichuan, China). During irradiation, unanesthetized mice were immobilized in a special jig and the right hind limb with tumor was put into the radiation field. A 5-mm-thick tissue equivalent bolus was used to direct the maximal radiation dose on the surface of the target tissue.

MTT assay. LLC cells were seeded in a 96-well plate and incubated for $24 \mathrm{~h}$, then treated with NS or Lip-mS (DNA at $1 \mu \mathrm{g} / \mathrm{ml})$. Forty-eight hours later, the cells were irradiated (2 Gy). After another 48 h, 3-(4,5-dimethylthiazol-2-yl)-2,5diphenyltetrazolium bromide (MTT) $(20 \mu 1,5 \mathrm{mg} / \mathrm{ml}$; Sigma) was added and it was further incubated for $4 \mathrm{~h}$ at $37^{\circ} \mathrm{C}$. Then the medium was removed from each well and DMSO (150 $\mu \mathrm{l})$ was added and incubated for $10 \mathrm{~min}$. Spectrometric absorbance at $540 \mathrm{~nm}$ was measured using a plate reader. The cell survival rate was calculated using the following equation: survival rate $=$ experimental group absorbance/control group absorbance x $100 \%$.

Flow cytometric analysis. LLC cells were seeded in a 6-well plate and incubated for $24 \mathrm{~h}$, then treated with NS or Lip-mS (DNA at $1 \mu \mathrm{g} / \mathrm{ml}$ ). Forty-eight hours later, the cells were irradiated (2 Gy). After another $48 \mathrm{~h}$, the cells were washed with PBS and resuspended in propidium iodide/RNase A solution $(0.5 \mathrm{ml})$, incubated at $37^{\circ} \mathrm{C}$ for $30 \mathrm{~min}$ and analyzed by flow cytometry.

Animal studies. All studies involving mice were approved by the Institute's Animal Care and Use Committee. Female C57BL/6 mice of 6 to 8 weeks old were purchased from experimental animal center of Sichuan University (Chengdu, Sichuan Province, China) and challenged s.c. with LLC cells ( $5 \times 10^{5}$ cells in $\left.50 \mu 1 \mathrm{PBS}\right)$ into the right hind limb. When the size of the tumors reached about $50 \mathrm{~mm}^{3}$, the mice were randomly divided into 4 groups (8 mice per group) and treated with NS, Lip-mS, radiation or Lip-mS + radiation, respectively. Lip-mS was injected into mice via the tail vein at $5 \mu \mathrm{g}$ per day once daily for 10 days (days 0 to 9) and the tumors were irradiated at 5 Gy per day for 4 days (days 1, 3, 6 and 8 ). Tumor size was determined by caliper measurement of the largest and perpendicular diameters every two days. Tumor volume was calculated according to the formula $\mathrm{V}=$ $0.52 \mathrm{ab}^{2}$, where $\mathrm{a}$ is the largest superficial diameter and $\mathrm{b}$ is the smallest superficial diameter.

Terminal deoxynucleotidyl transferase-mediated dUTP nick end-labeling (TUNEL) assay. The mice were sacrificed by cervical dislocation on day 14 after the initiation of Lip-mS administration. Primary tumors were excised, fixed in $10 \%$ neutral-buffered formalin solution and embedded in paraffin. Contiguous 3-5 $\mu \mathrm{m}$ sections were mounted. In order to highlight the cells that were undergoing apoptosis, unstained sections mounted in silanized slides were subjected to fluorescent in situ terminal deoxynucleotidyl transferasemediated dUTP nick end-labeling (TUNEL) assay by using an 


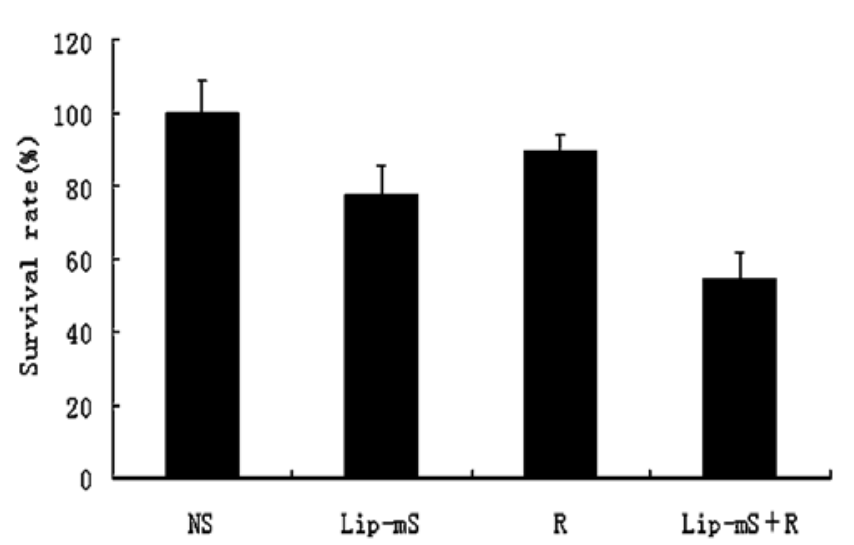

Figure 1. Growth inhibition in LLC cells by treatment with Lip-mS and radiation. LLC cells were treated with NS, Lip-mS (DNA at $1 \mu \mathrm{g} / \mathrm{ml}$ ), radiation $(2 \mathrm{~Gy})$, or Lip-mS + radiation. Growth inhibition was measured by the MTT assay. The proportion of surviving cells was calculated as a percentage of the control and data were represented as the mean $\pm \mathrm{SE}$. According to the results of the MTT assays, the combined treatment $(54.6 \%)$ significantly reduces LLC cell viability compared with the Lip-mS $(77.5 \%)$ or radiation $(89.6 \%)$ treatment alone $(\mathrm{P}<0.05)$.

in situ apoptotic cell detection kit (Promega, Madison WI, USA), according to the manufacturer's protocol. Representative images were taken under a light microscope (x200) in randomly selected fields.

CD31 immunohistochemical evaluation. Immunohistochemistry analyses of microvessel expression were done with goat anti-mouse CD31 antibody (Santa Cruz Biotechnology) using the labeled streptavidin-biotin method. That is to say, sections were deparaffinized in xylol and rehydrated in graded alcohol series. Antigen retrieval was carried out by autoclaving sections in retrieval buffer $(10 \mathrm{mM}$ pH 6.0 EDTA citrate buffer) for $3 \mathrm{~min}$ in saturated steam after up-pressure gaining $\left(126^{\circ} \mathrm{C}, 1.6\right.$ bars, $\left.23 \mathrm{psi}\right)$. Endogenous peroxidase activity was blocked by incubation in $3 \%$ hydrogen peroxide at room temperature free of light for $20 \mathrm{~min}$. Non-specific binding of reagents was quenched by incubation of sections for $20 \mathrm{~min}$ in 5\% normal rabbit serum. Sections were then incubated with goat anti-mouse CD31 (dilution 1/200) antibodies overnight at $4{ }^{\circ} \mathrm{C}$, followed by incubating with biotinylated rabbit antigoat IgG and then streptavidin-biotin-horseradish peroxidase complex at $37^{\circ} \mathrm{C}$ for $1 \mathrm{~h}$, respectively. Negative control was included with each run by substituting the primary antibody with non-immune rabbit serum. Cellular nuclei were counterstained with ameliorative Gill's hematoxylin. Representative images were taken under a light microscope (x400) in randomly selected fields.

Statistical analysis. In order to determine statistical significance, Student's t-test for non-paired data was performed. A value of $\mathrm{P}<0.05$ was considered to be statistically significant.

\section{Results}

Enhancement of the anti-tumor effect of radiation in vitro. In order to test the combined effect of Lip-mS with radiation in vitro, we treated LLC cells with NS, Lip-mS (DNA at $1 \mu \mathrm{g} / \mathrm{ml}$ ), radiation (2 Gy) or Lip-mS + radiation. Growth inhibition was analyzed by measuring cell viability with MTT assay. According to the results of the MTT assays, the combined treatment $(54.6 \%)$ significantly reduces LLC cell viability compared with the Lip-mS $(77.5 \%)$ or radiation $(89.6 \%)$ treatment alone $(\mathrm{P}<0.05)$ (Fig. 1$)$.

We also used flow cytometric analysis to evaluate the effect of Lip-mS and radiation on the induction of apoptosis in LLC cells. Lip-mS + radiation treatment $(36.2 \%)$ significantly increased the proportion of sub-G1 cells (apoptotic cells) compared with the other treatments (NS, 3.0\%; Lip-mS, 21.9\%; radiation, 9.6\%) (Fig. 2).

Moreover, the interactive anti-tumor effect of the combined treatment was greater than additive when compared with the expected effect of the combined treatment in vitro. The
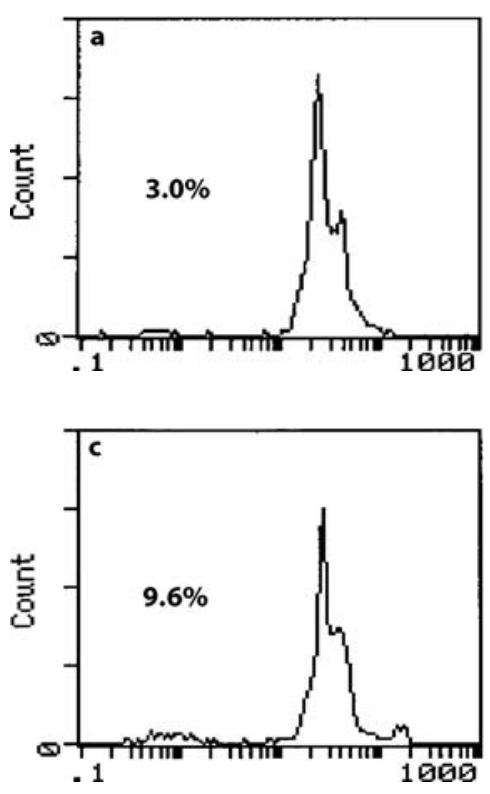
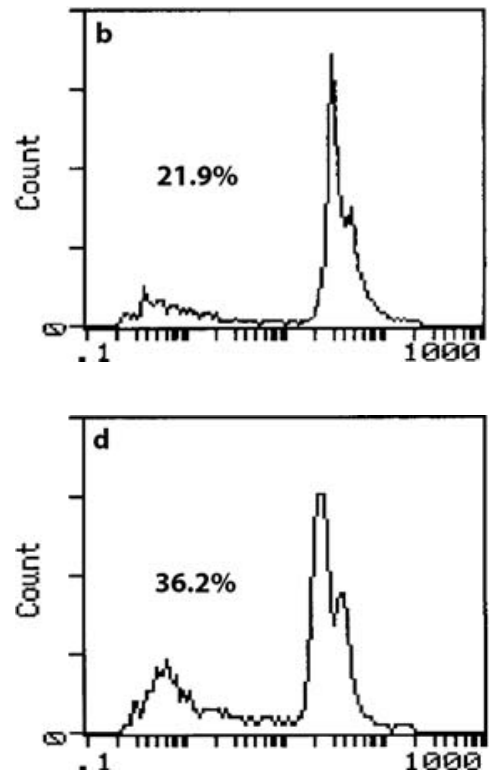

Figure 2. Induction of apoptosis in LLC cells by treatment with Lip-mS and radiation. LLC cells were treated with NS (a), Lip-mS (b), radiation (c), or Lip-mS + radiation (d). Flow cytometric analysis revealed the proportion of sub-G1 cells (apoptotic cells) to be $3.0 \%$ (a), $21.9 \%$ (b), $9.6 \%$ (c) and $36.2 \%$ (d), respectively. 


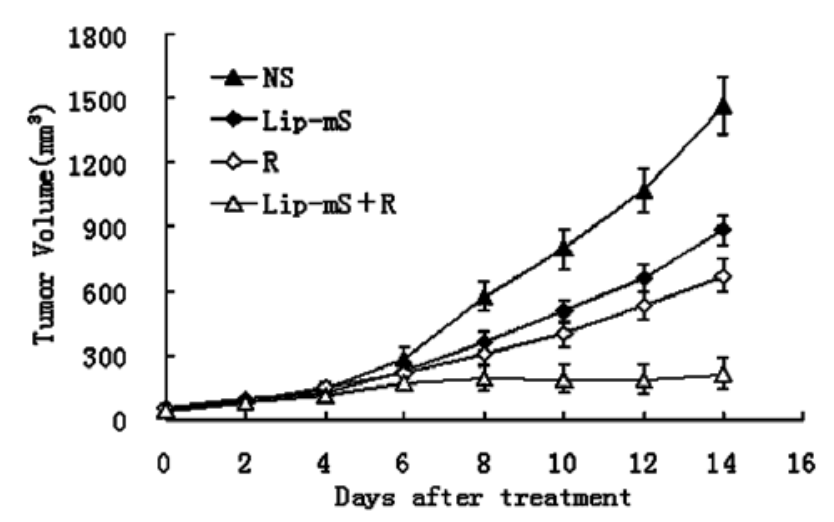

Figure 3. Lip-mS enhanced the anti-tumor effect of radiation in vivo. Mice bearing LLC tumors were treated with NS $(\Delta)$, Lip-mS $(\bullet)$, radiation $(\diamond)$ or Lip-mS + radiation $(\triangle)$. The combined treatment significantly reduced the mean tumor volume on day 14 when compared with the Lip-mS or radiation treatment group $(\mathrm{P}<0.05)$.

expected combined apoptotic effect was defined as follows: expected combined effect $=$ Lip $-\mathrm{mS}$ effect + radiation effect - Lip-mS effect x radiation effect. The Lip-mS and radiation treatment can induce apoptosis 21.9 and $9.6 \%$, respectively; thus, the expected induction of apoptosis in the combined treatment should be $29.4 \%$. However, the actual induction of apoptosis in the combined treatment is $36.2 \%$, suggesting greater than additive treatment effect.

Enhancement of the anti-tumor effect of radiation in vivo. The anti-tumor effect of Lip-mS in combination with radiation was assessed in mice bearing LLC tumors. The tumor growth curves demonstrated that both Lip-mS and radiation individually resulted in effective suppression of tumor growth, while the combined treatment had a superior anti-tumor effect when compared with the Lip-mS or radiation treatment alone $(\mathrm{P}<0.05)$ (Fig. 3). Moreover, the interactive anti-tumor effect of the combined treatment was also greater than their additive effect when compared with the expected effect of the combined treatment. On day 14 after the initiation of Lip-mS administration, the mean tumor volume of radiation group was $670.2 \mathrm{~mm}^{3}$, this represents a $54.3 \%$ volumetric reduction. Compared with the $39.6 \%$ reduction in tumor size observed in the Lip-mS treatment group, the expected volumetric reduction in tumor size of the combined treatment group should be $72.4 \%$. However, the percent reduction in the combined treatment group is $85.5 \%$, suggesting the greater than additive effect of the combined treatment.

In order to test by which possible mechanisms Lip-mS enhanced the anti-tumor effect of radiation in vivo, tumor sections of each group were stained with TUNEL reagent and anti-CD31 antibody to evaluate apoptosis rate and microvessel density as described above. An apparent increase in the number of apoptotic cells was observed within the tumors treated with the combination of Lip-mS and radiation compared with the other treatments (Fig. 4). Tumors of the NS and radiation treatment groups demonstrated high microvessel density, while those of the Lip-mS alone and combined treatment groups had decreased values (Fig. 5). These data suggest Lip-mS can cause the increased apoptosis of tumor cells and inhibition of tumor angiogenesis, which may play important roles in the enhancement of the anti-tumor effect of radiation in vivo.

\section{Discussion}

Radiotherapy is one of the most widely used treatments for cancer, with over half of all cancer patients receiving radiation
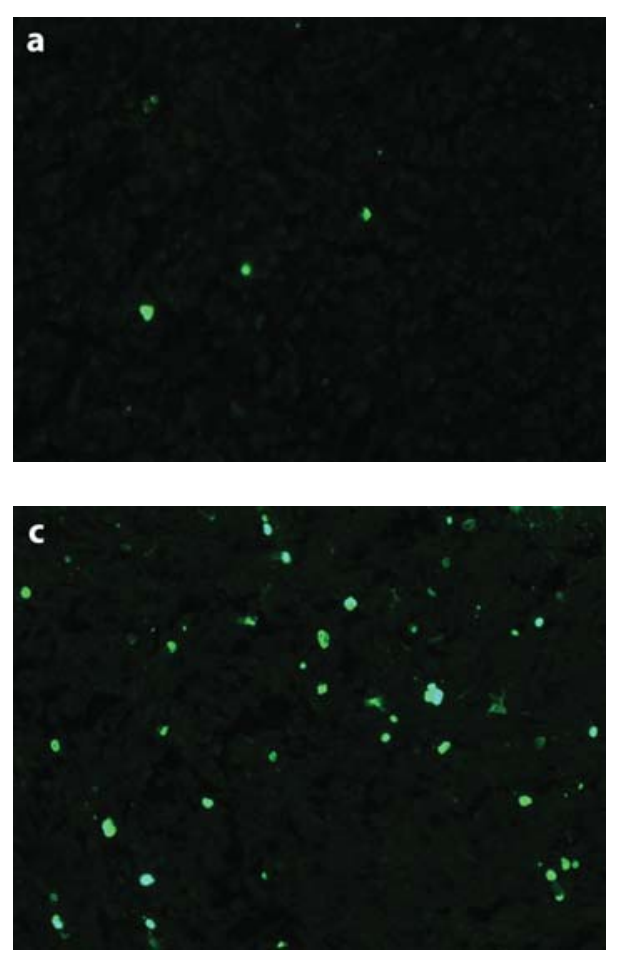
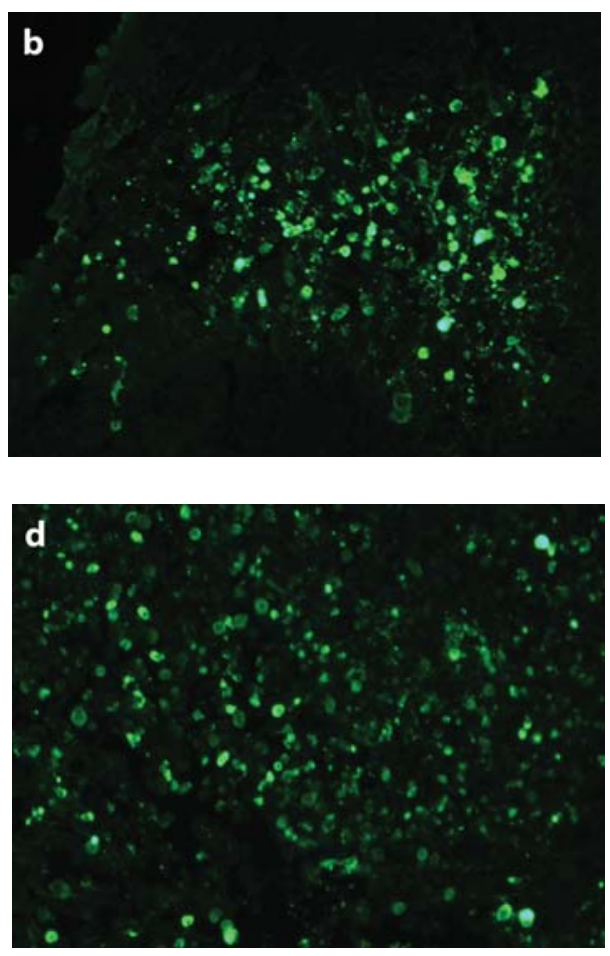

Figure 4. Combination of Lip-mS and radiation enhanced the induction of apoptosis in vivo. Tissue sections from tumor-bearing mice treated with NS (a), Lip-mS (b), radiation (c), or Lip-mS + radiation (d) were stained with FITC-DUTP. An apparent increase in the number of apoptotic cells was observed within tumors treated with a combination of Lip-mS and radiation compared with the other treatments. 

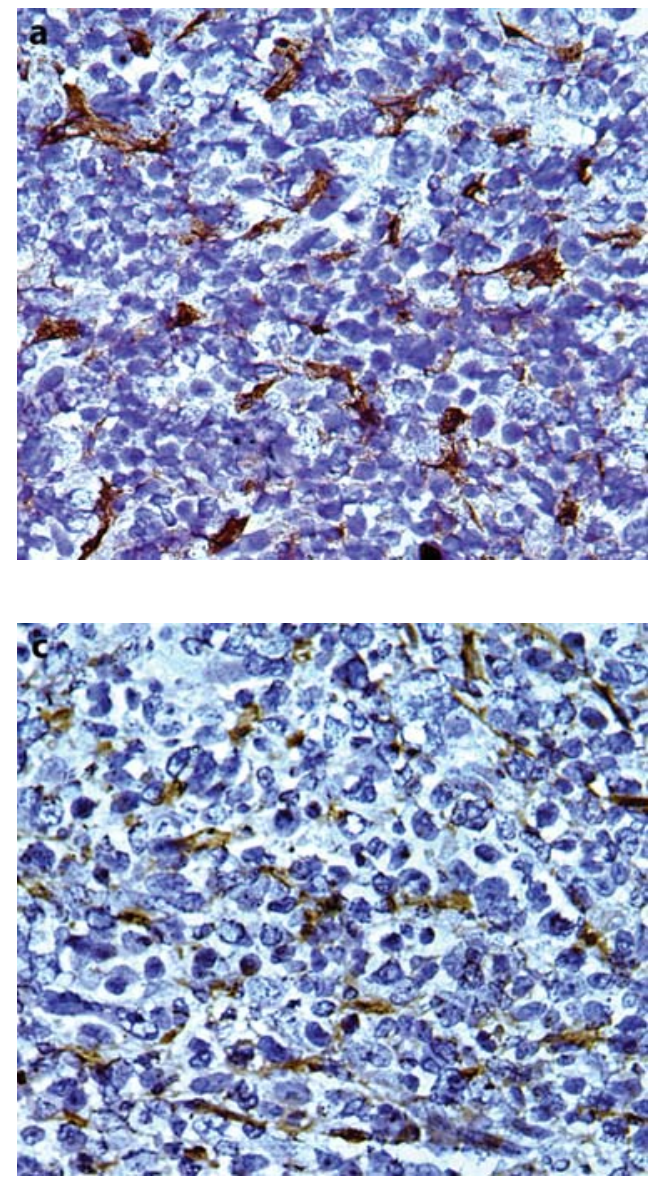
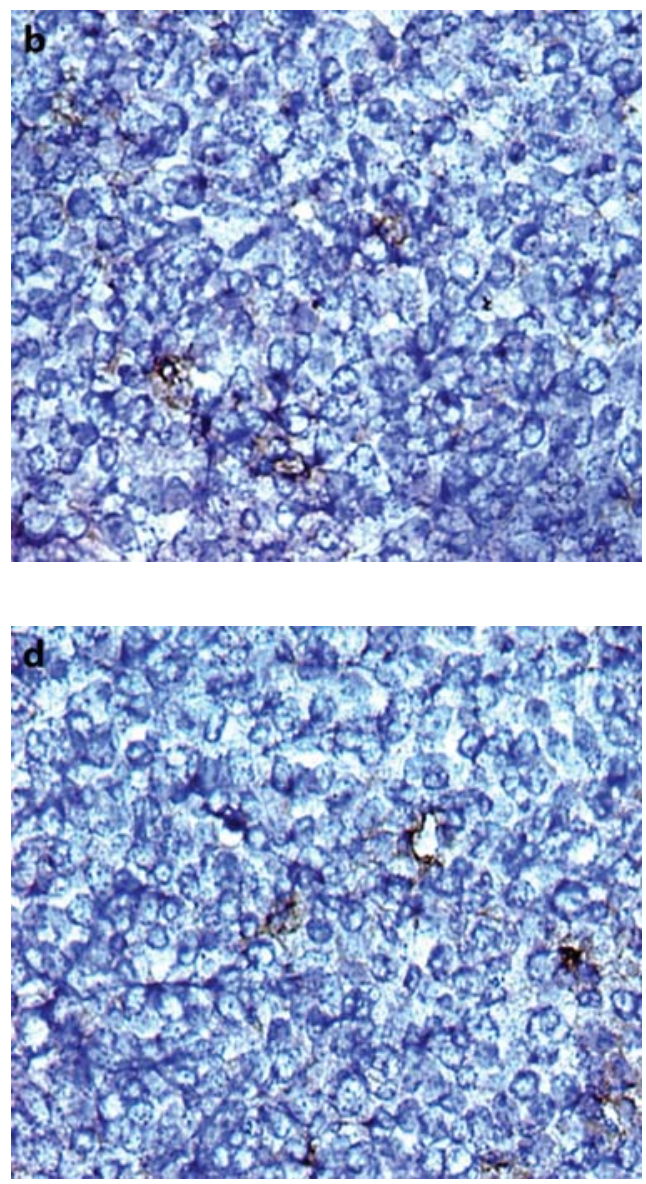

Figure 5. Inhibition of intratumoral angiogenesis assayed by CD31 staining of microvessel. Vascularization within tumors was detected by an antibody to CD31, representative images were taken under a light microscope (x400) in randomly selected fields. Tumors of the NS (a) and radiation (c) treatment groups demonstrated high microvessel density, while those of the Lip-mS (b) and Lip-mS + radiation (d) treatment groups showed apparent inhibition of angiogenesis.

at some point during their course of treatment (19), but to varying degrees of success, some tumors are highly responsive to radiation while others are typically very radioresistant and tend to progress even after high radiation doses (20), thereby increasing the radiosensitivity of tumor cells has the potential to significantly improve the rate of recovery from many malignancies. Radiosensitivity is due to a complex interaction of various factors (21), previously, it was reported that survivin also acts as a constitutive and inducible radio-resistance factor in a panel of tumor cells (4) and approaches designed to inhibit survivin expression or function may lead to tumor sensitisation to chemical and physical agents (22).

In the present study, the combination of Lip-mS gene therapy and radiation significantly enhanced the anti-tumor effect of radiation. Moreover, the interactive anti-tumor effect of the combined treatment was greater than their additive effect when compared with the expected effect of the combined treatment. These data suggest that inhibition of survivin using a dominant-negative mutant, survivin T34A, can sensitize LLC cells to radiation efficiently. The exact mechanism of interaction between survivin targeting agents and radiation, although remained to be determined, may be in part involved in three aspects as follows: increasing the apoptosis of tumor cells, inhibiting tumor angiogenesis and inducing a tumorprotective immune response.

Apoptosis, an evolutionarily conserved and genetically regulated process, preserves tissue and organ homeostasis by eliminating senescent or damaged cells (23). Aberrations of apoptosis are known to contribute to a variety of diseases including cancer and play an important role in tumor initiation, progression, and radioresistance (24). One cellular mechanism common to radiation is to kill tumor cells via apoptosis $(25,26)$. Radiation can directly damage DNA, resulting in mitochondrial damage due to the breakdown of the mitochondrial membrane potential, which will stimulate the initiation of apoptosis by releasing cytochrome c (27-29). Alternatively, radiation may directly damage the cellular membrane, resulting in the release of ceramide. Once released into the cytoplasm, ceramide can directly damage the mitochondrial membrane and then stimulate the release of cytochrome c (30). Released from mitochondria, cytochrome c can bind and activate the apoptotic protease-activating factor-1 (Apaf-1), causing activation of caspase- 9 within the apoptosome complex and leading to a protease cascade ultimately to apoptosis $(31,32)$. In this process of apoptosis, activation of caspases, a common step downstream of mitochondrial cytochrome c release, may be controlled by the intracellular IAP family (33).

As a structurally unique member of the IAP family, survivin plays an important role in the suppression of apoptosis by either directly or indirectly interfering with the function of caspases, the downstream effectors of apoptosis. XIAP, another IAP family member which contains three Baculovirus IAP Repeat (BIR) domains, can inhibit caspase- 3 and -7 by a linker region between the first two BIR domains and can also bind 
and inhibit caspase-9 via its third BIR (BIR3) domain. While survivin contains only one BIR domain, which appears in three-dimensional structure closely related to the BIR3 domain of XIAP, suggesting the possibility of binding and inhibitting caspase-9 directly $(34,35)$. Smac/Diablo, released from mitochondria into the cytosol along with cytochrome c, can increase Apo-2L/TRAIL-induced caspase-3 activity and downregulate the activity of IAPs during execution of the mitochondrial apoptosis pathway. Survivin may bind and sequester Smac/DIABLO, thus preventing Smac/DIABLO binding to other IAPs (36). Survivin has also been shown to interact with $\mathrm{Cdk} 4$, which releases p21 to complex with and inactivate caspase-3 (37). Therefore it is not difficult to understand that inactivation of IAPs such as survivin, as a barrier to apoptosis, should be possible to reverse malignant cell radioresistance, effectively promote the apoptotic signals conveyed by radiation and ultimately drive tumor cells into the apoptotic suicide process.

Enhancement of tumor response to radiation by survivin targeting agents has also been explained by a decrease in tumor angiogenesis after treatment. To supply oxygen and other nutrients, angiogenesis plays an important role in the growth and progression of solid tumors (38). Survivin is also overexpressed in endothelial cells of the tumor vasculature during the proliferative and remodeling phases of angiogenesis and generates a cytoprotective mechanism for these cells (39). Moreover, radiation may induce tumor cells to secrete protective cytokines, such as VEGF, which could further upregulate survivin expression, inhibit apoptosis and enhance radioresistance in vascular endothelial cells (40). These findings identify survivin as a novel protective gene expressed by endothelial cells during angiogenesis, suggesting that survivin targeting may be able to induce apoptosis in endothelial cells and sensitise the tumor vasculature to different anti-cancer modalities including radiation. In the previous and present studies (16), we have demonstrated that inhibition of survivin by a T34A dominant-negative mutant significantly increased apoptosis of endothelial cells and reduced tumor-associated angiogenesis, which may retard or prevent adequate nourishment of tumors, result in tumor growth stasis and therefore is complementary to the anti-tumor effect of radiation.

As a strategy for cancer therapy, anti-angiogenic therapy attempts to stop new vessels from forming around a tumor and break up the existing network of abnormal capillaries that feeds the cancerous mass (41), but it is well known that oxygen is a potent radiosensitizer, the combination of anti-angiogenic targeting agents with radiation appears to be a counterintuitive strategy because a reduction in tumor vasculature would be expected to reduce blood perfusion and oxygen concentration, so to result in hypoxia in tumor cells which is resistant to radiotherapy (42). However, accumulating experimental evidence revealed that anti-angiogenic agents may actually increase oxygen levels, reduce tumor hypoxia and provide a rationale for combining these agents with radiation $(43,44)$. Tumor oxygenation is a function of perfusion and oxygen consumption, anti-angiogenic agents may increase perfusion by reducing the number of immature and inefficient vessels, and reduce the overall consumption by reducing the number of oxygen-consuming tumor cells and endothelial cells, all of which give rise to more oxygenated tumor cells that are sensitive to radiation (45).

Survivin is also an attractive target for novel immunotherapies against cancer (46). Previously, the immunological properties of survivin were demonstrated by the detection of survivin-reactive antibodies (47) and cytolytic T-cell clones $(48,49)$. In particular, the survivin-based DNA vaccine can inhibit tumor growth and metastases by evoking an effective $\mathrm{T}$ cell-mediated immune response, which simultaneously attacks both the tumor and endothelial cells of tumor vessels, thereby triggering tumor cell apoptosis and suppression of angiogenesis (50) and eventually rendering tumor cells more sensitive to the anti-tumor effect of radiation.

Many attempts have been undertaken in order to understand the mechanisms of radiosensitivity of tumors, our data suggest that a dominant-negative mutant, survivin T34A, could sensitize LLC cells to radiation efficiently and the synergistic anti-tumor activity may in part result from increasing the apoptosis of tumor cells, inhibiting tumor angiogenesis and inducing a tumor-protective immune response in the combined treatment. These findings may serve as a basis for the rational design of future strategies and it appears to be only a matter of time before our knowledge of the mechanisms of radiosensitivity of tumors can be merged and applied to improve the clinical results of tumor radiotherapy.

\section{Acknowledgements}

This study was in part supported by the National 863 Project of China 2007AA021201 and partly by the Postdoctoral Science Fund of China 20070410390.

\section{References}

1. Tanaka K, Iwamoto S, Gon G, Nohara T, Iwamoto M and Tanigawa N: Expression of survivin and its relationship to loss of apoptosis in breast carcinomas. Clin Cancer Res 6: 127-134, 2000

2. Miller M, Smith D, Windsor A and Kessling A: Survivin gene expression and prognosis in recurrent colorectal cancer. Gut 48: 137-138, 2001.

3. Sarela AI, Verbeke CS, Ramsdale J, Davies CL, Markham AF and Guillou PJ: Expression of survivin, a novel inhibitor of apoptosis and cell cycle regulatory protein, in pancreatic adenocarcinoma. Br J Cancer 86: 886-892, 2002.

4. Asanuma K, Moriai R, Yajima T, Yagihashi A, Yamada M, Kobayashi D and Watanabe N: Survivin as a radioresistance factor in pancreatic cancer. Jpn J Cancer Res 91: 1204-1209, 2000.

5. Colella G, Zaffaroni N, Pennati M, Benini E and Daidone MG: Expression of the anti-apoptosis gene survivin correlates with taxol resistance in human ovarian cancer. Proc Am Assoc Cancer Res 42: 302, 2001.

6. Ling X, Bernacki RJ, Brattain MG and Li F: Induction of survivin expression by taxol (paclitaxel) is an early event, which is independent of taxol-mediated G2/M arrest. J Biol Chem 279: 15196-15203, 2004.

7. Altieri DC: Validating survivin as a cancer therapeutic target. Nat Cancer Rev 3: 46-54, 2003.

8. Pennati M, Folini M and Zaffaroni N: Targeting survivin in cancer therapy: fulfilled promises and open questions. Carcinogenesis 28 : 1133-1139, 2007.

9. Kanwar JR, Shen WP, Kanwar RK, Berg RW and Krissansen GW: Effects of survivin antagonists on growth of established tumors and B7-1 immunogene therapy. J Natl Cancer Inst 93: 1541-1552, 2001.

10. Pennati M, Colella G, Folini M, Citti L, Daidone MG and Zaffaroni N: Ribozyme-mediated attenuation of survivin expression sensitizes human melanoma cells to cisplatin-induced apoptosis. J Clin Invest 109: 285-286, 2002. 
11. Paduano F, Villa R, Pennati M, Folini M, Binda M, Daidone MG and Zaffaroni N: Silencing of survivin gene by small interfering RNAs produces supra-additive growth suppression in combination with 17-allylamino-17-demethoxygeldanamycin in human prostate cancer cells. Mol Cancer Ther 5: 179-186, 2006.

12. Jiang G, Li J, Zeng Z and Xian L: Lentivirus-mediated gene therapy by suppressing survivin in BALB/c nude mice bearing oral squamous cell carcinoma. Cancer Biol Ther 5: 435-440, 2006.

13. Pisarev V, Yu B, Salup R, Sherman S and Gabrilovich DI: Full-length dominant-negative survivin for cancer immunotherapy. Clin Cancer Res 9: 6523-6533, 2003.

14. Grossman D, Kim PJ, Schechner JS and Altieri DC: Inhibition of melanoma tumor growth in vivo by survivin targeting. Proc Natl Acad Sci USA 98: 635-640, 2001.

15. McKay TR, Bell S, Tenev T, Stoll V, Lopes R, Lemoine NR and McNeish IA: Procaspase 3 expression in ovarian carcinoma cells increases survivin transcription which can be countered with a dominant-negative mutant, survivin T34A; a combination gene therapy strategy. Oncogene 22: 3539-3547, 2003.

16. Peng XC, Yang L, Wei YQ, et al: Efficient inhibition of murine breast cancer growth and metastasis by gene transferred mouse survivin Thr34 $\rightarrow$ Ala mutant. J Exp Clin Cancer Res 27: 46, 2008.

17. Templeton NS, Lasic DD, Frederik PM, Strey HH, Roberts DD and Pavlakis GN: Improved DNA:liposome complexes for increased systemic delivery and gene expression. Nat Biotech 15 647-652, 1997

18. Lin X, Chen X, Zhao X, et al: Efficient inhibition of intraperitoneal human ovarian cancer growth and prolonged survival by gene transfer of vesicular stomatitis virus matrix protein in nude mice. Gynecol Oncol 104: 540-546, 2007.

19. Owen JB, Coia LR and Hanks GE: Recent patterns of growth in radiation therapy facilities in the United States: a patterns of care study report. Int J Radiat Oncol Biol Phys 24: 983-986, 1992.

20. Jung $M$ and Dritschilo A: Signal transduction and cellular responses to ionizing radiation. Semin Radiat Oncol 6: 268-272, 1996.

21. Zhivotovsky B, Joseph B and Orrenius S: Tumor radiosensitivity and apoptosis. Exp Cell Res 248: 10-17, 1999.

22. Zaffaroni $\mathrm{N}$ and Daidone MG: Survivin expression and resistance to anticancer treatments: perspectives for new therapeutic interventions. Drug Resistance Updates 5: 65-72, 2002.

23. Vaux DL and Korsmeyer SJ: Cell death in development. Cell 96: 245-254, 1999

24. Hanahan D and Weinberg RA: The hallmarks of cancer. Cell 100: 57-70, 2000.

25. Baierlein SA, Distel L, Sieber R, Weiss C, Rödel C, Sauer R and Rödel F: Combined effect of tumor necrosis factor-alpha and ionizing radiation on the induction of apoptosis in 5637 bladder carcinoma cells. Strahlenther Onkol 182: 467-472, 2006.

26. Weiss C, Rödel F, Wolf I, et al: Combined-modality treatment and organ preservation in bladder cancer. Do molecular markers predict outcome? Strahlenther Onkol 181: 213-222, 2005.

27. Nakano K and Vousden KH: PUMA, a novel proapoptotic gene, is induced by p53. Mol Cell 7: 683-694,2001.

28. Oda E, Ohki R, Murasawa H, et al: Noxa, a BH3-only member of the Bcl-2 family and candidate mediator of p53-induced apoptosis. Science 288: 1053-1058, 2000.

29. Wei MC, Zong WX, Cheng EH, et al: Proapoptotic BAX and BAK: a requisite gateway to mitochondrial dysfunction and death. Science 292: 727-730, 2001

30. Haimovitz-Friedman A, Kan CC, Ehleiter D, Persaud RS, McLoughlin M, Fuks Z and Kolesnick RN: Ionizing radiation acts on cellularmembranes to generate ceramide and initiate apoptosis. J Exp Med 180: 525-535, 1994.
31. Cryns V and Yuan Y: Proteases to die for. Genes Dev 12: 1551-1570, 1999.

32. Thornberry NA and Lazebnik Y: Caspases: enemies within. Science 281: 1312-1316, 1998

33. Deveraux QL and Reed JC: IAP family proteins: suppressors of apoptosis. Genes Dev 13: 239-252, 1999.

34. Sun C, Cai M, Gunasekera AH, et al: NMR structure and mutagenesis of the inhibitor-of-apoptosis protein XIAP. Nature 401: 818-822, 1999.

35. Shi Y: Survivin structure: crystal unclear. Nat Struct Biol 7: 620-623, 2000.

36. Du C, Fang M, Li Y, Li L and Wang X: Smac, a mitochondrial protein that promotes cytochrome c-dependent caspase activation by eliminating IAP inhibition. Cell 102: 33-42, 2000.

37. Suzuki A, Hayashida M, Ito T, et al: Survivin initiates cell cycle entry by the competitive interaction with Cdk4/p16 (INK4a) and Cdk2/cyclin E complex activation. Oncogene 19: 3225-3234, 2000 .

38. Hanahan D and Folkman J: Patterns and emerging mechanisms of the angiogenic switch during tumorigenesis. Cell 86: 353-364, 1996.

39. O'Connor DS, Schechner JS, Adida C, et al: Control of apoptosis during angiogenesis by survivin expression in endothelial cells. Am J Pathol 156: 393-398, 2000.

40. Fuks Z and Kolesnick R: Engaging the vascular component of the tumor response. Cancer Cell 8: 89-91, 2005.

41. Marx J: Angiogenesis: a boost for tumor starvation. Science 301: 452-454, 2003.

42. Thomlinson RH and Gray LH: The histological structure of some human lung cancers and the possible implications for radiotherapy. Br J Cancer 9: 539-549, 1955.

43. Griffin RJ, Williams BW, Wild R, Cherrington JM, Park H and Song CW: Simultaneous inhibition of the receptor kinase activity of vascular endothelial, fibroblast, and platelet-derived growth factors suppresses tumor growth and enhances tumor radiation response. Cancer Res 62: 1702-1706, 2002.

44. Lee CG, Heijn M, di Tomaso E, et al: Anti-vascular endothelial growth factor treatment augments tumor radiation response under normoxic or hypoxic conditions. Cancer Res 60: 5565-5570, 2000.

45. Eberhard A, Kahlert S, Goede V, Hemmerlein B, Plate KH and Augustin HG: Heterogeneity of angiogenesis and blood vessel maturation in human tumors: implications for antiangiogenic tumor therapies. Cancer Res 60: 1388-1393, 2000.

46. Andersen MH and Thor SP: Survivin - a universal tumor antigen. Histol Histopathol 17: 669-675, 2002.

47. Rohayem J, Diestelkoetter P, Weigle B, et al: Antibody response to the tumor-associated inhibitor of apoptosis protein in cancer patients. Cancer Res 60: 1815-1817, 2000.

48. Schmidt SM, Schag K, Müller MR, et al: Survivin is a shared tumor-associated antigen expressed in a broad variety of malignancies and recognized by specific cytotoxic T cells. Blood 102: 571-576, 2003

49. Reker S, Meier A, Holten-Andersen L, Svane IM, Becker JC, thor Straten P and Andersen MH: Identification of novel survivin-derived CTL epitopes. Cancer Biol Ther 3: 173-179, 2004.

50. Xiang R, Mizutani N, Luo Y, et al: A DNA vaccine targeting survivin combines apoptosis with suppression of angiogenesis in lung tumor eradication. Cancer Res 65: 553-561, 2005. 